\title{
Excitation of highly charged hydrogen-like ions by the impact of equivelocity electrons and protons: a comparative study
}

\author{
B. Najjari ${ }^{1}$ and A.B.Voitkiv ${ }^{2}$ \\ 1 Institut Pluridisciplinaire Hubert Curien, Université de Strasbourg, \\ 23 rue du Loess, BP 28, 67037 Strasbourg Cedex 2, France * \\ 2 Max-Planck-Institut für Kernphysik, Saupfercheckweg 1, D-69117 Heidelberg, Germany
}

\begin{abstract}
We consider excitation of highly charged hydrogen-like ions by the impact of equivelocity electrons and protons. The kinetic energy of the protons is more than three orders of magnitude larger than that of the equivelocity electrons. It is shown, however, that despite this fact, the electrons can be much more effective in inducing excitation at collision velocities (slightly) above the theshold for electron impact excitation. The basic reason for this is the strong distortion of the motion of the electron by the attractive field of the nucleus of the highly charged ion.
\end{abstract}

PACS numbers: PACS:34.10.+x, 34.50.Fa

\section{INTRODUCTION}

Excitation of highly charged ions by the impact of charged particles (projectiles) is an interesting physical process which may also have many applications. In particular, in case of electron projectiles the studies of this process are of importance for the physics of hightemperature plasmas produced in laboratories and existing in astrophysical sources.

Collisions of energetic highly charged ions with atoms represent an important field of research at modern accelerators of heavy ions. In collisions of a highly charged ion with neutral atoms the ion can also be excited. If the momentum transferred to the atom in the collision is much larger than the typical momenta of the electrons in the atom the excitation process can be regarded as occurring due to the incoherent interactions of the electron of the ion with the nucleus and the electrons of the atom which behave with respect to each other as (quasi-) free particles [1]. In the rest frame of the ion the excitation then can be viewed as induced by the incoherent impacts of the "independent" beams of the atomic nucleus and atomic electrons.

If the collision velocity is much larger than the Bohr velocity in the $K$-shell of the atom, the contribution $\sigma_{N}$ to the excitation cross section caused by the interaction with the nucleus of the atom is very simply related to the cross section $\sigma_{p}$ for excitation by proton impact: $\sigma_{N}=$ $Z_{A}^{2} \sigma_{p}$, where $Z_{A}$ is the charge of the atomic nucleus.

We thus see that the excitation of a highly charged ion in collisions with atoms can, under certain conditions, be reduced to two basic processes: excitations in collisions with an equivelocity electron and proton. In this respect a question arises about the relative effectiveness of these two types of projectiles in producing the excitation. Note that althought excitation of ions by electronic

*Electronic address: bennaceur.najjari@iphc.cnrs.fr

${ }^{\dagger}$ Electronic address: alexander.voitkiv@mpi-hd.mpg.de and protonic (nuclei) projectiles has been studied (see e.g. [2]-[11] and references therein), to our knowledge these studies were always done separately for electrons and protons.

From the perspective of atomic physics the differences between the electrons and protons mainly include i) the huge difference in masses and also ii) the opposite sign of their charges. For instance, due to the first point, the kinetic energies of equivelocity electrons and protons differ by about a factor of 2000 .

A trivial consequence of this fact is that in the range of impact velocities $v$ below the threshold velocity $v_{t h}$ for incident electrons, where these electrons do not have enough energy to excite the ion, the protons do have and are capable of producing excitation. Besides, it is also quite natural to expect that at sufficiently high impact velocities, where the kinetic energy of the incident electron is much larger than the excitation energy of the ion, the cross sections for excitation by equivelocity electrons and protons will converge.

What, however, can can say about the relative effectiveness of these two projectiles in case when the impact velocity is already above the threshold velocity $v_{t h}$ but the kinetic energy of the incident electron is not yet much larger than the excitation energy? Below in this article, where excitation of highly charged hydrogen-like ions by the impacts of equivelocity electrons and protons is considered for a broad range of the atomic numbers of the ions, we shall address this question.

The article is organized as follows. In the next section we briefly outline the basic physics of proton-ion and electron-ion collisions and discuss how one can calculate the corresponding excitation cross sections. In section III we present results for excitation cross sections of hydrogen-like ions of nickel, xenon, erbium, bismuth and uranium (the corresponding atomic numbers are: $Z_{I}=28,54,68,83$ and 92$)$. The main results are summarized in section IV.

Atomic units $\left(\hbar=m_{e}=|e|=1\right)$ are used throughout the paper except where otherwise stated. 


\section{THEORY}

To an excellent approximation the nucleus of the ion, which has a charge $Z_{i}\left(Z_{i} \gg 1\right)$, can be regarded as infinitely heavy and the field which it creates as an external field. We shall consider the collisions in the rest frame of this nucleus and choose its position as the origin.

The transition amplitude for the excitation of the ion by the projectile (an electron or a proton) can be written according to (see, e.g. [11])

$$
S_{f i}=-\frac{i}{c^{2}} \int d^{4} x \int d^{4} y j_{\mu}(x) D^{\mu \nu}(x-y) J_{\nu}(y) .
$$

Here, $j_{\mu}(x)$ and $J_{\nu}(y)(\mu, \nu=0,1,2,3)$ are the electromagnetic transition 4 -current densities generated by the electron of the ion at a space-time point $x$ and by the projectile at a space-time point $y$, respectively, and $D^{\mu \nu}(x-y)$ is the propagator of the electromagnetic field which transmits the interaction between these particles. The contravariant $a^{\mu}$ and covariant $a_{\mu}$ 4-vectors are given by $a^{\mu}=\left(a^{0}, \mathbf{a}\right)$ and $a_{\mu}=\left(a^{0},-\mathbf{a}\right)$. The metric tensor $g_{\mu \nu}$ of the four-dimensional space is defined by $g_{00}=-g_{11}=-g_{22}=-g_{33}=1$ and $g_{\mu \nu}=0$ for $\mu \neq \nu$. In (11) the summation over the repeated greek indices is implied.

Provided the transition currents in (11) (and in the corresponding exchange contribution to the amplitude) are evaluated using the relativistic description of the bound and free particles, the treatment of the excitation process is fully relativistic. In particular, in this treatment there is no upper limit on the collision energy and also excitation of most heavy ions may be considered.

A. The effect of the field of the nucleus of the ion on the motion of the incident and scattered particle

Since we suppose that the nucleus of the ion has a high charge, its field can in general strongly influence not only the motion of the bound electron but also that of the incident (and scattered) particle. A simple estimate for the magnitude of the effect of this field on the motion of the incident electron and/or proton in the process of excitation can be obtained in the following way [1]. Assume that there is a particle with a charge $q$ and mass $m$ which is incident with a velocity $v$ on the nucleus $Z_{i}$. One can estimate the effect of the field by using the ratio $\varsigma=\delta p / p_{i}$, where $p_{i}=m \gamma v\left(\gamma=1 / \sqrt{1-v^{2} / c^{2}}\right)$ is the initial momentum of the incident particle and $\delta p$ is the change in the momentum of this particle caused by the field of $Z_{i}$. This change is roughly given by $\delta p \sim Z_{i} q /(b v)$, where $b$ is the impact parameter. For the problem of excitation the typical impact parameters are of the order of $1 / Z_{i}$ or larger. Therefore,

$$
\varsigma \simeq \frac{|q|}{m \gamma} \frac{Z_{i}^{2}}{v^{2}}
$$

In order to make the process of excitation in collisions with electrons energetically possible, one roughly needs $Z_{i}^{2} / m_{e} v^{2} \lesssim 1$. Therefore, it follows from (2) that for the impact velocities of interest for the present article the parameter $\varsigma$ is very small in the case of proton projectiles $\left(\varsigma \lesssim 1 / m_{p} \gamma<10^{-3}\right)$. As a result, the field of the ionic nucleus does not affect the motion of the proton which can be regarded in the initial and final states as a free particle. In contrast, for electron projectiles $\varsigma$ may be close to 1 ( $\lesssim 1 / m_{e} \gamma \lesssim 1$ ) which means that the field of the nucleus can very strongly distort the motion of the electron. Indeed, it will be seen below that this distortion can have a crucial impact on the process of excitation.

\section{B. Excitation in collisions with protons}

The treatment of excitation of a highly charged hydrogen-like ion by protons is based on the following main points (see e.g. [1]).

First, the charge of the proton is much smaller than that of the highly charged nucleus of the ion. As a result, the interaction between the proton and the electron of the ion in the process of excitation is much weaker than the interaction between the electron and the ionic nucleus. Therefore, it can be regarded as a weak perturbation and may be taken into account within one-photon exchange (first-order perturbation theory).

Second, as was already mentioned in the previous subsection, due to the relatively heavy mass of the proton the distortion of its motion caused by the field of the nucleus of the ion can be ignored. Then, regarding the proton as a Dirac particle, one can approximate the initial and final states of the proton by (Dirac) plane-waves.

\section{Excitation in collisions with electrons}

Let us now briefly discuss the treatment of excitation of a highly charged hydrogen-like ion in collisions with electrons (see e.g. [2]-[5], [1]).

Like in the case of collisions with protons, the interaction between the incident electron and the electron of the ion is comparatively very weak. Therefore, the description of this interaction in the excitation process can be reduced to just single-photon exchange between the electrons.

However, in contrast to the excitation by protons, the interaction between the incident electron and the ion in general cannot be treated within the first-order perturbation theory. The reason is that the motion of the incident (and scattered) electron can be very substantially affected by its interaction with the nucleus of the ion. This point can be addressed by describing not only the bound but also the continuum electron as moving in the Coulomb field of the nucleus of the ion. 
Further, the electrons are indistinguishable and, therefore, the exchange effect has to be taken into account by including an additional diagram (the so called exchange diagram) into the treatment of electron-impact excitation.

Below we shall refer to the treatment, which (i) describes the continuum electron as moving in the Coulomb field of the nucleus of the ion, (ii) takes into account the interaction between the continuum and bound electrons within first-order perturbation theory and (iii) includes the exchange effect, as Approach I.

In addition, in the next section we shall present results for electron impact excitation obtained by using another - simplified - treatment. This simple treatment - termed Approach II - also describes the interaction between the free and bound electrons within first-order perturbation theory but neglects the distortion of the continuum electron states by the field of the ion (approximating them by Dirac plane waves) and does not take into account the exchange effect between the free and bound electrons.

Note that both these approaches do not take into account the channel of resonance excitation. This channel may become effective when the energy of the initial configuration of the electrons (the incident electron plus the electron bound in the ground state of a hydrogenlike ion) closely matches an energy of a doubly excited bound state of the correspoding helium-like ion. Under such conditions the excitation of a hydrogen-like ion may proceed via formation of a doubly excited bound state of the corresponding helium-like ion which then decays due to autoionization into an excited state of the hydrogenlike ion (see e.g. [7]).

\section{NUMERICAL RESULTS AND DISCUSSION}

Here we shall consider excitation of $\mathrm{Ni}^{27+}(1 \mathrm{~s})$, $\mathrm{Xe}^{53+}(1 \mathrm{~s}), \quad \mathrm{Er}^{67+}(1 \mathrm{~s}), \quad \mathrm{Bi}^{82+}(1 \mathrm{~s})$ and $\mathrm{U}^{91+}(1 \mathrm{~s})$ ions caused by the impacts of equivelocity electrons and protons. We restrict ourselves to the excitation into the $L$-shell only (for which the cross sections are much larger than for the higher shells). The corresponding results are shown in figures 1-5 where the calculated cross sections for excitation by electron impact are displayed by solid and dash curves and those for excitation by protons are depicted by dot curves.

The main conclusion, which can be drawn from the figures, is that, despite the huge difference in kinetic energy, the electrons are overally not less effective than protons in inducing the excitation. One more important point following from the figures is that the relative effectiveness of the electron projectiles compared to that of the protons substantially increases when the atomic number of the ion grows.

The very large difference in kinetic energies between equivelocity electrons and protons makes the phase space for the final states of the outgoing electron (the cross section is proportional to the volume of this space) much

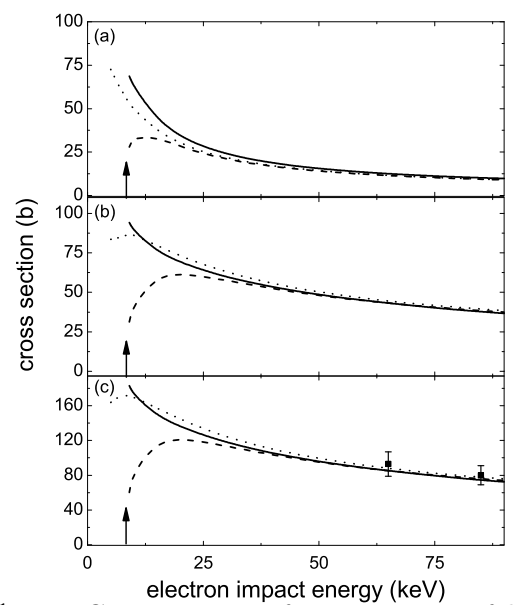

FIG. 1: Cross sections for excitation of hydrogen-like nickel $\left(Z_{I}=28\right)$ by equivelocity electrons and protons given as a function of the electron kinetic energy. Sections (a), (b) and (c) show the cross sections for the $1 s_{1 / 2} \rightarrow 2 s_{1 / 2}, 1 s_{1 / 2} \rightarrow 2 p_{1 / 2}$ and $1 s_{1 / 2}$ $\rightarrow 2 p_{3 / 2}$ transitions respectively. Solid and dash curves show the results for excitation by electron impact obtained by using Approach I and Approach II, respectively. Dot curve displays the results for excitation by protons. Experimental data for electron impact excitation are from [8].

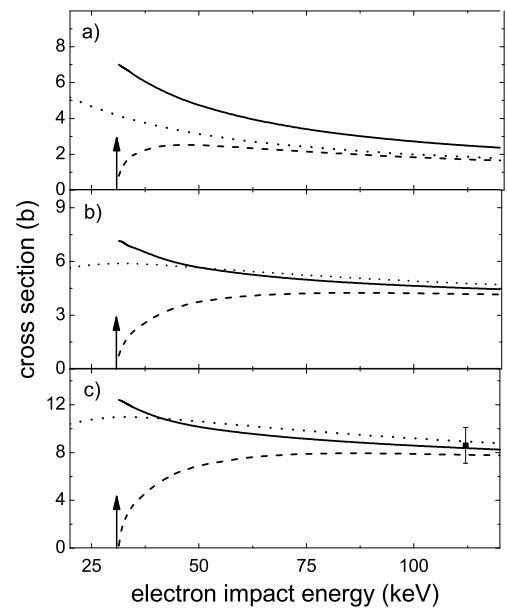

FIG. 2: $\quad$ Same as in figure 1 but for excitation of hydrogenlike xenon $\left(Z_{I}=54\right)$. Experimental result for electron impact excitation is from [6].

smaller compared to that of the scattered proton. Since the volume of this space is proportional to $\sim k_{f}^{2} d k_{f} \sim$ $k_{f} \varepsilon_{f} d \varepsilon_{f} / c^{2}$, where $k_{f}$ and $\varepsilon_{f}$ are the momentum and total energy of the outgoing electron, it becomes especially small when the impact energy of the incident electron approaches the excitation threshold.

This is why the cross section calculated using the simplified Approach II, in which the incident and scattered electron is described by plane waves, increases from zero at the excitation threshold. Contrary to Approach II, however, the more sophisticated Approach I leads to the cross sections which have their maxima at the electron impact energy equal to the excitation energy [12]. 


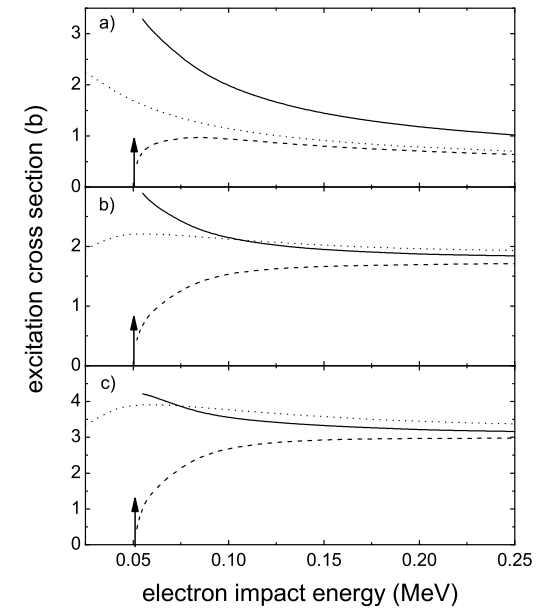

FIG. 3: Same as in figure 1 but for excitation of hydrogen-like erbium $\left(Z_{I}=68\right)$.

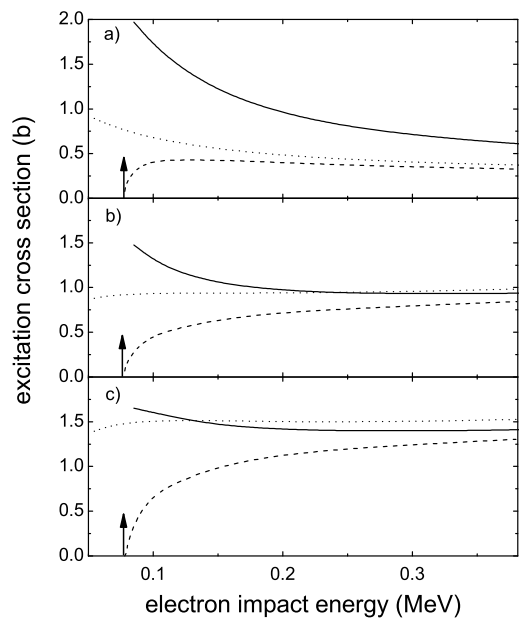

FIG. 4: $\quad$ Same as in figure 1 but for excitation of hydrogen-like bismuth $\left(Z_{I}=83\right)$.

Such a behaviour is the consequence of the well known singularity which is present for the continuum states of an electron moving in an attractive Coulomb field with an asymptotic momentum $k \rightarrow 0$. This singularity compensates for the smallness of the phase space of the outgoing electron. Thus, it is the distortion of the motion of the unbound electron by the attractive Coulomb field of the ion which makes the electronic projectiles so effective in exciting the ion. This distortion is especially strong for the low-velocity electrons which results in the fact that at the excitation threshold and slighly above it the electrons can be even much more effective than the equivelocity protons.

\section{CONCLUSION}

We have considered excitation of highly charged hydrogen-like ions in collisions with equivelocity electrons

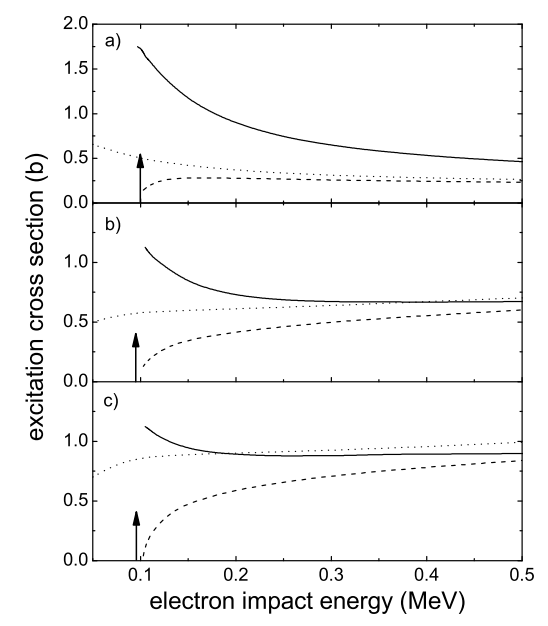

FIG. 5: Same as in figure 1 but for excitation of hydrogen-like uranium $\left(Z_{I}=92\right)$.

and protons. We have shown that the electronic projectiles are not less effective in inducing the excitation than the protons. Moreover, according to our results the relative effectiveness of electronic projectiles increases when the atomic number of the ion increases.

The differences between these two types of projectiles, which influence the process of excitation, lie in the very large differences in their masses and also in the sign of charge.

The large mass of protonic projectiles in general favours the process of excitation. Indeed, it furnishes a large phase space for the scattered proton and also strongly diminishes the effect of the repulsion between the proton and the nucleus of the ion enabling the proton to come closer to the electron of the ion (compared, say, to an equivelocity positron) increasing their interaction.

The small mass of electronic projectile has a two-fold influence on the excitation process. One the one hand, compared to a proton an equivelocity electron has much less kinetic energy which per se would make the electrons substantially less effective in inducing excitation close to the threshold compared to equivelocity protons. However, due to the smallness of the electron mass the motion of the incident and scattered electrons may be very strongly affected by the field of the nucleus of the ion. For electrons this field is attractive and pulls in the incident electron closer to the electron of the ion which increases the chances for excitation.

Based on the results of this study one can also make a (rather obvious) conclusion that at the threshold a positron projectile would be very inefficient in inducing the excitation because of its strong repulsion by the nucleus. In particular, in collisions with positronium the effect of excitation at velocities slighly above $v_{t h}$ would fully come from the electron while the positron would be merely a spectator. In this respect it is interesting to note that such a situation seems to take place even 
in collisions of a positronium with a neutral atom 13

where the repulsion effect is much weaker than in case of collisions with a highly charged ion.

\section{Acknowledgement}

A.B.V. acknowledges the support from the Extreme Matter Institute EMMI.

[1] B.Najjari and A.B.Voitkiv, Phys. Rev. A 85052712 (2012)

[2] C.J.Fontes, D.H.Sampson and H.L.Zhang, Phys. Rev. A 471009 (1993).

[3] C.J.Fontes, D.H.Sampson and H.L.Zhang, Phys. Rev. A 493704 (1994).

[4] C.J.Fontes, D.H.Sampson and H.L.Zhang, Phys. Rev. A 51 R12 (1995).

[5] D.L.Moors and K.J.Reed, Phys. Rev. A 51 R9 (1995).

[6] K.Widmann et al, Am. Inst. Phys. Conf. Proc. 506, 444 (2000).

[7] A. Müller, Advances in At., Mol. and Opt. Phys. 55, 293 (2008)

[8] D.B.Thorn, P.Beiersdorfer, G.V.Brown, R.L.Kelley, C.A.Kilbourne and F.S.Porter, Journal of Physics: Con- ference Series 163, 012036 (2009).

[9] J.Eichler and W.E.Meyerhof, Relativistic Atomic Collisions, Academic Press, New York (1995).

[10] A.B.Voitkiv, B.Najjari and J.Ullrich, Phys. Rev. A 75 062716 (2007).

[11] A.B.Voitkiv and J.Ullrich, Relativistic Collisions of Structured Atomic Particles (Springer-Verlag, Berlin, 2008); A.B.Voitkiv, Phys.Rep. 392191 (2004).

[12] For instance, for the $1 s_{1 / 2} \rightarrow 2 s_{1 / 2}$ cross section at the threshold Approach I yields (approximately) 75, 7.2, 3.5, 2.1 and $1.7 \mathrm{~b}$ for hydrogen-like $\mathrm{Ni}, \mathrm{Xe}, \mathrm{Er}, \mathrm{Bi}$ and $\mathrm{U}$, respectively.

[13] S.J.Brawley et al, Science 330789 (2010); Phys. Rev. Lett. 105263401 (2010). 